\title{
Liderazgo para una gestión moderna de procesos educativos
}

\section{Leadership management for modern educational processes}

\author{
Guiselle María Garbanzo Vargas \\ Directora de la Escuela de Administración Educativa \\ Universidad de Costa Rica \\ San José, Costa Rica \\ gmgarban@gmail.com \\ Victor Hugo Orozco Delgado \\ Docente de la Escuela de Administración Educativa \\ Universidad de Costa Rica \\ San José, Costa Rica \\ vhorode@hotmail.com
}

Recibido 11-11-2009 • Aceptado 28-02-2010 • Corregido 24-03-2010

\section{Contextualización}

Resumen: La autora y el autor realizan a manera de ensayo una contextualización social sobre la que actúa la educación resaltando en ella la complejidad social en la que se desarrolla el proceso educativo y los retos que le demanda la sociedad de la información. Desde este contexto se replantea el papel que una gestión moderna sustentada en un liderazgo pertinente representa en el logro de los objetivos y fines educativos que la sociedad se plantea. Se replantean las características personales y de liderazgo que en una gestión moderna de la educación se requiere, así como factores claves de la calidad de la gestión de la educación. Se desarrollan los elementos de liderazgo aplicados a la educación.

Palabras clave: Liderazgo, liderazgo en las organizaciones educativas, administración de la educación, gestión de la educación, administración y organizaciones educativas.
En el actual contexto mundial, la sociedad ha sido testigo de diferentes acontecimientos de trascendencia histórica que han transformado la actividad del ser humano, caracterizada por la revolución tecnológica, sobre la cual Castells (2002) considera que está centrada en torno a las tecnologías de la información y a su vez modifica la base material de la sociedad en forma acelerada. Estos acontecimientos se han manifestado con mayor intensidad en los últimos veinte años. Fenómenos políticos, económicos y ambientales figuran entre los más importantes que han marcado un nuevo orden social.

Entre los fenómenos políticos de mayor relevancia sobresalen la caída del 


\begin{abstract}
The authors'essay on social contextualization considers education, highlighting the social complexity of educational development and challenges when society demands information. Within this context, the role of modern management and leadership is redefined and its relationship to the achievement of society's educational objectives and aims. Personal characteristics and leadership practices of modern educational management are reconsidered, as well as key quality factors of educational management. The components of educational leadership are developed.
\end{abstract}

Key words: Leadership, educational leadership, educational administration, educational management, educational administration and educational organizations.
Muro de Berlín, la desintegración de la Unión Soviética, el desvanecimiento del movimiento comunista a escala mundial y los cambios profundos que experimenta el capitalismo, lo que se manifiesta en una mayor flexibilidad en la gestión y tanto la descentralización así como en la interconexión de las distintas empresas. También llaman la atención la creación de la Comunidad de la Unión Europea y la emergencia de gobiernos de tendencia izquierdista en Suramérica, entre los que se encuentran países como Venezuela, Ecuador, Bolivia y Argentina.

En el campo económico, el mundo ha experimentado una histórica y significativa recesión, en la que las economías asumen interdependencia y subyace una nueva relación entre el modelo económico, el Estado y la sociedad; todo ello bajo una interacción versátil. El Estado abandona un rol benefactor pasando a un modelo económico liberal. El Pacífico Asiático ha sobresalido por un destacado ascenso industrial predominante a escala global. La unificación de la comunidad económica europea también asume un papel estratégico en el orden económico. A las organizaciones, dentro de sus parámetros de competitividad, les ha correspondido un papel más flexible e interconectado y descentralizado, en el que el individualismo se incrementa cada día como estructura social; a su vez, las sociedades han pasado a funcionar en forma interdependiente, donde el tiempo real no es un límite y la virtualidad emerge como un orden organizacional Castells (2002).

En lo que respecta a los acontecimientos ambientales, que a escala mundial son ampliamente reconocidos tanto por su importancia como por sus implicaciones de la sobrevivencia de la especie humana en el Orbe, se encuentra la carencia de otras alternativas energéticas sustitutas del petróleo debido a la extinción segura de este preciado líquido en un mediano plazo; también está el fenómeno del calentamiento global, causado por las enormes cantidades de dióxido de carbono producido por la 
actividad humana, lo que ha hecho que las temperaturas de los océanos se eleven peligrosamente (Gore, 2007).

Castells (2002) hace referencia a algunos de los acontecimientos que han marcado la contemporaneidad, entre los que sobresalen:

- Aumento de poder del capital frente al trabajo remunerado.

- Intervención del Estado para regular los mercados selectivamente.

- Desmantelamiento del Estado Benefactor.

- Intensificación de la competitividad económica global.

- Integración global de los mercados.

- El Pacífico Asiático como nuevo eje industrial global predominante.

- Unificación de la comunidad económica de Europa.

- Unificación y desintegración del antiguo tercer mundo.

- La transformación gradual de Rusia.

- Economías a escala mundial que funcionan como unidad en tiempo real.

- Revolución tecnológica.

- Inserción masiva de la mujer en el mundo laboral.

Ante estos acontecimientos, Castells (2002) considera que se ha dado una acentuación del desarrollo en forma desigual, donde se observa tanto la liberación paralela de fuerzas productivas de la revolución de la información así como, en forma simultánea, la consolidación de la miseria humana. Todo esto no ha hecho otra cosa que cambiar el orden social, para lo que Hargreaves, citado por Aguerrondo, Lugo y Rossi (2001, p. 17): consideran que “... la flexibilidad económica, la mundialización de la economía, la comprensión del espacio y el tiempo, la sociedad digital, el hogar digital, el teletrabajo, son realidades cada vez más presentes". Estas realidades conducen a un mundo más competitivo, de cambios profundos en cualquier campo que se mire.
Esos cambios profundos a los que se hacen referencia son incontenibles y de gran impacto en múltiples escenarios, lo que implica entre otras cosas, que rápidamente quedan obsoletos conocimientos y prácticas asumidas como válidas en los distintos campos de las actividades humanas. Los efectos no son otros que buscar nuevas pautas de desarrollo y competitividad para las organizaciones, donde las personas que las integran son su prioridad. Todo esto emerge en la denominada hoy "sociedad de la información", ante la cual, la educación asume un papel estratégico y las organizaciones educativas necesitan ser conducidas mediante un liderazgo eficaz.

\section{La sociedad de la información y las organizaciones educativas}

Autores comos Castells (2002) ubican el surgimiento de la sociedad de la información en la década de 1970. Druker, citado por Castells (2002), desde un inicio advirtió que la nueva forma de trabajar tenía que ver con la información y que este cambio de paradigma abriría paso de una sociedad industrial a una sociedad del conocimiento.

Philippe Quéau, Director de la División Sociedad de la Información de la UNESCO, citado por Menezes (2000), considera que no se puede comparar el conocimiento con otros productos, puesto que este posee propiedades específicas que lo hacen incomparable, se propaga rápidamente sin costo alguno y sus retribuciones pueden ser altas o ningunas; además, es un modo de pensar, no un producto, y tiene un impacto social y político. Este autor considera que la brecha digital es social y económica.

La revolución de la información es una revolución del conocimiento, es la reorganización del trabajo tradicional mediante la aplicación del conocimiento y en especial mediante el análisis en forma sistemática y lógica. La clave para 
mantener el liderazgo en la economía es la ciencia cognitiva, depende de la posición social que tengan los profesionales del conocimiento y la aceptación social de sus valores (Drucker, 1999 en Micheli, 2002 citado por Crovi, 2002).

La sociedad del conocimiento, como se le denomina hoy, necesita sistemas educativos con capacidad para aprender y desarrollar nuevas competencias. En este campo, las organizaciones educativas se vuelven trascendentales, un adecuado liderazgo tras ellas las posiciona en un contexto de alta competitividad. El ser humano es un ser organizacional y son los individuos quienes conducen las organizaciones. Los individuos que integran las organizaciones lo hacen con diferentes niveles de formación académica, distintas características personales y variadas posiciones organizacionales. A su vez, las organizaciones se estructuran en distintas modalidades y atienden diferentes programas educativos.

El sistema educativo concreta su proyecto educativo mediante las organizaciones educativas distribuidas en diferentes niveles, a saber: preescolar, primaria, secundaria y educación universitaria; eso sí, se requiere de un acertado liderazgo que promueva las condiciones de autodesarrollo profesional en forma competitiva como única respuesta a las demandas de por sí exigentes del contexto en el que a la educación hoy le ha correspondido actuar; relaciones laborales/organizacionales de por sí complejas. Necesariamente, las organizaciones educativas requieren redefinir su rol, de manera que ofrezcan formación de calidad incorporando las adaptaciones necesarias ante el entorno para que la educación se vincule de forma acertada con las necesidades nacionales, y de esta forma se transforme en un instrumento eficaz de cohesión y desarrollo social.

En este escenario, los cambios económicos y realidades productivas y sociales demandan nuevos compromisos educativos, así como líderes competitivos capaces de transformar la educación acertadamente según los requerimientos de los tiempos. Un adecuado proceso de planificación es una condición para propiciar un cambio de paradigma, pasando de una educación basada en la transmisión y memorización a una que se enfatice en los procesos de comprensión de competencias que involucran tanto el saber, como el saber hacer y el saber ser. Esto es posible siempre y cuando las organizaciones educativas marchen en armonía con los objetivos por los cuales se crearon, sin perder de vista que su fin último es la formación integral del ser humano, tal y como está explicito en los fines de la educación costarricense:

a) La formación de ciudadanos amantes de su Patria, conscientes de sus deberes, de sus derechos y de sus libertades fundamentales, con profundo sentido de responsabilidad y de respeto a la dignidad humana;

b) Contribuir al desenvolvimiento pleno de la personalidad humana;

c) Formar ciudadanos para una democracia en que se concilien los intereses del individuo con los de la comunidad;

e) Conservar y ampliar la herencia cultural, impartiendo conocimientos sobre la historia del hombre, las grandes obras de la literatura y los conceptos filosóficos fundamentales. Artículo 2. Ley Fundamental de Educación. (Costa Rica, Ministerio de Educación Pública, 2006, p. 49)

Partiendo de estos nobles ideales, como son los fines de la educación costarricense, por los cuales se debe luchar de forma incansable desde las organizaciones educativas indistintamente de su posición, modalidad y nivel en el sistema educativo costarricense y, tomando en cuenta la complejidad y los cambios acelerados actuales, quienes asumen las responsabilidad de conducir las organizaciones educativas se convierten en elementos estratégicos. Estas personas son las responsables de liderar procesos de gestión que permitan a las organizaciones educativas concretar su proyecto curricular para alcanzar así los fines y objetivos de la educación, y enfrentar con éxito los desafíos a los cuales se expone la educación constantemente. Todo ello es posible bajo un adecuado liderazgo organizacional, 
alejado de los paradigmas tradicionales, que coloque la educación según los estándares de la época.

\section{Organizaciones educativas y liderazgo organizacional}

El desarrollo de las organizaciones está vinculado al liderazgo que se ejerza en ellas. Las organizaciones educativas necesitan de un acertado liderazgo para rediseñar modelos de mayor autonomía institucional y gestión moderna de procesos en cada contexto específico, de manera que permita a las organizaciones no solo el fortalecimiento de sus ofertas académicas, sino también los espacios concretos de toma de decisiones que posibiliten la combinación adecuada de tiempos, espacios, programas y potencial humano, y con ello cumplir con las expectativas que hoy día se tienen de las organizaciones educativas.

Las organizaciones educativas son los espacios donde se concreta el proceso enseñanza-aprendizaje que, además de desarrollar las capacidades cognitivas del estudiantado, potencializa la formación integral, donde la ética, estética y el componente cultural y espiritual subyacen en una relación armoniosa con un paradigma epistemológico responsable. Dada su complejidad, el liderazgo mediante el cual se gestan las organizaciones educativas debe trascender de un corte tradicional a un liderazgo renovador.

En las organizaciones las personas no actúan en forma aislada; más bien, es mediante las interacciones con otras personas que pueden alcanzar los objetivos. Es una relación donde la influencia es recíproca, puesto que precisamente las limitaciones de cada individuo se articulan gracias a la cooperación de los integrantes de la organización. Las organizaciones son sistemas de cooperación, son sistemas sociales (Chiavenato, 2004). En el caso de las organizaciones educativas, la interacción, la disposición y la finalidad de alcanzar los fines y objetivos de la educación, es lo que la hace existir y requerir de un líder que la conduzca, de manera que el proyecto educativo se concrete según los requerimientos emanados.

Hay que tener presente que las organizaciones educativas se caracterizan por un conjunto de actividades conscientemente conducidas, donde la cooperación que se produzca principalmente es motivada por el gestor; en este caso, quien ocupa el liderazgo de la organización. Una condición importante para su desarrollo es el nivel de autonomía en la toma de decisiones que el sistema educativo le otorgue al gestor de la educación.

Este planteamiento coincide con un grupo de directores y directoras de centros educativos del país quienes participaron en un estudio denominado "Necesidades profesionales y de capacitación de los administradores educativos para responder a las demandas del siglo XXI", realizado por Garbanzo (2004), en el que estos profesionales plantean algunas de las necesidades más sentidas en el campo de la Administración de la Educación, las que consideran fundamentales para responder a los desafíos del siglo XXI, tal y como se indican en la tabla 1 (ver tabla 1).

Además de estas necesidades profesionales y de formación que requieren los profesionales en el campo de la Administración de la Educación, Garbanzo y Orozco (2007) consideran que son muchos los desafíos a los que se enfrenta la educación costarricense hoy; en su mayoría, estos desafíos se han manifestado a lo largo de la historia de la educación en diferentes niveles de profundidad. El Informe del Estado de la Educación, publicado en el 2005 y fundamentado con datos del 2004 (CONARE/ Programa Estado de la Educación en Desarrollo Humano Sostenible, 2005) demostró ante la sociedad costarricense $\mathrm{y}$ apoyado en distintos macroindicadores, diversos factores de interés nacional que de una $u$ otra forma han estado siempre 
Tabla 1

Necesidades profesionales de capacitación y formación de los administradores de la educación para responder a los desafíos del siglo XXI

\begin{tabular}{|c|c|}
\hline Necesidades profesionales en capacitación & Necesidades en la formación académica \\
\hline $\begin{array}{l}\text { - Legislación educativa } \\
\text { - Conocimiento de herramientas } \\
\text { de computación. } \\
\text { - Indicadores de calidad educativa. } \\
\text { - } \text { Formación ética y moral } \\
\text { - Conocimilidades para relacionarse con la comunidad } \\
\text { curriculares }\end{array}$ & $\begin{array}{l}\text { - Utilización de tecnología en informática } \\
\text { - } \quad \text { Conocimientos actualizados en administración } \\
\text { - } \quad \text { Formacativa } \\
\text { - } \quad \text { Legislación ética y moral. } \\
\text { - } \quad \text { Gestión del currículo } \\
\text { - } \quad \text { Rescate de valores } \\
\text { - } \quad \text { Liderazgo académico }\end{array}$ \\
\hline
\end{tabular}

Fuente: Elaboración propia.

presentes en el país. Estos desafíos tienen relación directa con la administración de las organizaciones educativas y son:

- universalizar la cobertura preescolar y secundaria,

- mejorar la calidad del sistema educativo,

- $\quad$ reducir la reprobación y mejorar la eficiencia,

- retener a los estudiantes y las estudiantes del sistema educativo,

- disminuir brechas socio-espaciales,

- ampliar la inversión en educación,

- mejorar las precarias condiciones de trabajo de las docentes y los docentes,

- mejorar la rectoría del sector,

- reforma institucional,

- fortalecer el sistema de monitoreo y evaluación,

- fortalecer la investigación educativa,

- reforzar el papel de las universidades públicas como agentes, de movilidad social,

- mejorar el monitoreo y el control de calidad de la enseñanza universitaria, $\mathrm{y}$

- fortalecer el vínculo entre la generación del conocimiento y su aplicación al desarrollo (CONARE/Programa Estado de la Nación en Desarrollo Humano Sostenible 2005).
El informe sostiene que importantes debilidades en la gestión del sistema educativo se constituyen en obstáculos para atender la problemática en el sistema como tal. Ante tales circunstancias, es impostergable para la educación costarricense que sus administradores de la educación, además de dinamizar las organizaciones que dirigen, promuevan la innovación y el cambio como herramientas estratégicas que permitan atender con éxito las desafiantes y complejas demandas del contexto, asegurándole así al sistema educativo mejores niveles de calidad educativa, lo cual es y debe ser una tarea permanente de la educación en sus diferentes ámbitos en respuesta a sus fines y objetivos. Para ello, es indispensable romper con el paradigma tradicional de la gestión de la educación y pasar a una gestión moderna de la educación, donde el liderazgo acertado, responsable, inteligente y comprometido con los fines de la educación sea el que prevalezca.

No se trata solo de abordar la problemática conceptual en torno a lo que se concibe como calidad de la educación, sino también diseñar modelos de gestión que propicien elevar la calidad educativa mediante su operacionalización. Estos modelos deben ser sostenibles y propositivos en forma constante y deben ser conducidos, eso sí, mediante un liderazgo 
participativo y pedagógico, que trascienda el contexto institucional, todo ello como condición para una gestión de calidad en la educación. Para estos efectos, entendiendo calidad de la educación como aquellos procesos educativos que bajo una adecuada conducción ofrecen las condiciones necesarias para que el conocimiento se imparta y se aprenda en forma adecuada; es decir, se concreten los propósitos de la educación costarricense bajo la conducción de una gestión de calidad.

\section{Gestión de calidad en educación}

El tránsito de la democracia representativa a una democracia participativa que se vive a escala nacional acentúa la necesidad de reformular las bases desde donde organizar y gestionar la educación. Una educación en la que los principios como dignidad humana, justicia social, igualdad de derechos, oportunidades, libertad, participación y transparencia, entre otros, constituyan la base sobre la que se gesten auténticos procesos educativos de calidad.

Calidad cuya concepción es compleja, sobre todo porque en el caso específico de la educación se refiere más a un servicio que a un producto, y a un servicio de formación cuyos resultados son a largo plazo, en el cual sus parámetros de calidad constantemente varían según las necesidades del contexto. Alrededor del concepto de calidad se dan tantas diversas concepciones como autores que lo analicen, los cuales se fundamentan en diferentes indicadores. Asimismo, la conceptualización del término considera también el ámbito y las condiciones educativas que se analicen. Antúnez (1999) plantea como criterios de gestión de calidad específicos en educación los siguientes:

- Recursos suficientes: capacidad de adecuar los recursos personales, materiales y funcionales a los fines que se pretenden y su uso eficaz y eficiente.

- Revisión y evaluación constante: capacidad de ejecutar el control interno, externo, técnico y social para llevar estas a cabo con la intención de utilizar sus resultados para mejorar la teoría y la práctica.

- Cauces y órganos para posibilitar la participación en la gestión de la institución.

- Dispositivos y acciones específicas para el desarrollo personal y profesional de los enseñantes, directivos y especialistas.

- Clima institucional satisfactorio y estimulante.

- Actuaciones concretas con relación a la atención hacia la diversidad de los alumnos y de los profesores.

- Autonomía suficiente en la institución, en sus directivos y en los enseñantes.

De igual manera, Braslavsky (2006) define algunos de los factores para una educación de calidad, en el presente siglo, que son importantes de considerar:

- Capacidad de conducción de los directores: hace referencia a la importancia del rol directivo como potencial gestor en las organizaciones educativas para promover aprendizajes de calidad.

- Trabajo en equipo: un aspecto importante a considerar en los procesos de gestión es el trabajo en equipo dentro de la escuela y de los sistemas educativos. Esto implica necesariamente cambiar tradiciones, por muchos años arraigadas en el quehacer diario; es indispensable trabajar en equipo en todos o cualquiera de los niveles posibles, esto constituye una clave en el proceso del logro de una educación de calidad.

Calidad y cantidad de recursos: la calidad y cantidad de recursos 
y materiales disponibles son otro factor a considerar; estos determinan condiciones de gestión.

Los elementos anteriormente planteados por Braslavsky (2006) constituyen, en alguna medida, pilares fundamentales sobre los cuales se construye una propuesta educativa renovada, razón de ser de quienes hoy día efectivamente desean dar el paso hacia una gestión de calidad de la educación, lo que viene a ser una condición impostergable.

En el mismo sentido, Posner, citada por Aguerrondo et al. (2001), propone algunas características de las escuelas de calidad:

- $\quad$ Equipo directivo centrado en el currículo, con clara hegemonía de lo pedagógico.

- $\quad$ Proyecto coherente que impregna y diseña la vida escolar, y perfila una cierta cultura interna.

- Clima institucional motivador hacia la totalidad de la comunidad educativa.

- Organización de espacios para compartir la experiencia profesional, que tiene como base un sistema de autoevaluación y autorregulación.

- Redes de comunicación y coordinación apoyadas en la fortaleza de los equipos de trabajo.

- Formación docente a partir de la práctica pedagógica.

Estos elementos evidencian la relevancia de la gestión de las organizaciones educativas. El rol del equipo de conducción como facilitador y gestor de procesos de desarrollo organizacional es determinante. Los docentes, por su parte, se convierten entonces en creadores y productores de la propuesta curricular con la conducción de una gestión moderna, de parte de los profesionales encargados de liderar la administración de la educación.

Es necesario, por lo tanto, que quienes dirigen centros educativos promuevan un estilo de liderazgo participativo, impulsor y coordinador de actuaciones que favorezcan climas de trabajo en donde se dinamice el proceso de participación, el debate y se flexibilice el cambio; todo ello como condición para una eficaz operacionalización de los propósitos de la educación. Se requiere un liderazgo que promueva la colaboración y sea compartido por equipos directivos o equipos de gestión que tengan gran influencia en la mejora global del proceso educativo.

El liderazgo es fundamental en la administración, el gestor necesita conocer cómo motivar y conducir las personas que integran la organización educativa, es una influencia interpersonal ejercida en un momento determinado, siempre orientado a la consecución de los objetivos propuestos, es un fenómeno social que tiene gran importancia en las interacciones humanas y en los fines y objetivos propuestos por las organizaciones, en este caso las educativas Chiavenato (2004). Al respecto, la escuela debe redireccionar su acción hacia la transmisión de prioridades claras, focalización de lo pedagógico, instalar prácticas de evaluación permanente y generar climas organizacionales positivos Aguerrondo et al. (2001).

Los anteriores elementos constituyen fundamentos teóricos que sustentan la base de una gestión moderna de procesos educativos que, en el caso costarricense, aporta los elementos necesarios para la transformación profunda de las formas de trabajo. Una gestión moderna que sitúe al sistema educativo en óptimas condiciones para avanzar hacia los objetivos estratégicos que lo están desafiando: calidad, equidad, inclusividad, pertinencia del currículo y profesionalización de las acciones educacionales. Ante este contexto, adquieren relevancia modelos de gestión educativa modernos que permitan el desarrollo educativo según las necesidades sociales acordes con la época. 


\section{Modelo de gestión educativa}

Muchas de las necesidades y problemas que enfrentan los profesionales que se ocupan de la administración de las organizaciones educativas en su ejercicio están relacionados con la concepción tradicional de la administración que se tenga, lo que le impide trascender más allá del "activismo" y el "cosismo" en administración, como en ocasiones se le designa.

Una gestión de la educación pertinente debe reunir, entre otros elementos, características esenciales de la personalidad y un liderazgo consolidado que tenga como norte la búsqueda permanente de logros. Entre las principales características de la personalidad se encuentran:

- temperamento equilibrado,

- tolerancia a la crítica,

- $\quad$ seguridad y capacidad de decisión,

- claridad a fin de evitar ambigüedades y confusión,

- actitud de reflexión y autocrítica,

- actitud democrática,

- congruencia con la actuación,

- facilidad de comunicación,

- conciencia de que cada institución determina sus ideas, valores, políticas y acciones,

- capacidad de implementar el liderazgo como ejercicio indispensable de influencia, con un alto nivel de convocatoria, $\mathrm{y}$

- $\quad$ ser ágil en la conducción de grupos (García, Rojas y Campos, 2002 y Alvarez, 1998).

implican:

Así mismo, estas capacidades

- delegar,

- tener la capacidad para organizar y animar equipos de trabajo eficaces,

- ser capaz de generar la circulación sistemática de la información, profesionalizar elroly enriquecerlocon los aportes del campo organizativo, conducir con coherencia los valores democráticos, estar capacitado para trabajar con ética y compromiso,

ejercer un liderazgo como ejercicio indispensable de influencia y capacidad convocante,

- poseer agilidad en la conducción de grupos, y

- tener conciencia de que cada institución determina su ideario-valores, políticas y acciones.

Sobre el liderazgo, se requiere:

- $\quad$ tener una visión clara y conocida por todos, expresada a través de su proyecto de dirección;

- $\quad$ traducir la visión en objetivos y altas expectativas;

- $\quad$ establecer un clima laboral que favorezca la consecución de los objetivos; supervisar y evaluar el progreso de sus colaboradores;

ser un ejemplo de trabajo duro y constante;

reconocer la singularidad de cada colaborador y desde ella hacerle crecer profesionalmente;

ser flexible y tener capacidad para el diálogo;

preocuparse más por las personas que por la rutina administrativa;

conocer las dinámicas internas de la organización y el liderazgo informal que permanece oculto;

ser tolerante con la ambigüedad y saber desenvolverse eficazmente en situaciones ambiguas;

abordar los problemas desde una perspectiva altamente analítica, buscando relaciones causa-efecto para aportar soluciones;

preocuparse más por las soluciones a los problemas que por los culpables; preferir comprometer a los colaboradores en la toma de decisiones a que ejecuten sumisamente las órdenes; 
- establecer sistemas de comunicación que permitan fluidez y transparencia;

- crear relaciones de amistad con su personal dejando clara la autoridad que le proporciona su liderazgo;

- $\quad$ poseer seguridad y un sentido muy desarrollado de sí mismo como individuo;

- $\quad$ ser amable, saber escuchar y tomar en cuenta los intereses, necesidades y ambiciones de sus colaboradores; y - tener claridad sobre el impacto de la toma de sus decisiones (Alvarez, 1998).

Todos estos elementos en forma articulada y coherente proporcionan condiciones para una gestión estratégica en educación, sustentada en un liderazgo pertinente, con altas posibilidades para lograr los objetivos organizacionales, que en el campo de la educación se caracterizan por su complejidad y variabilidad en sus modalidades y niveles, según las exigencias sociales.

\section{Liderazgo para una gestión estratégica en educación}

En el marco de acción del sistema educativo costarricense, existe la tendencia a utilizar indistintamente los términos dirección, liderazgo y gestión cuando se hace referencia a la administración de centros educativos. Claro está como lo plantean Pascual e Immegart, citados por Borrell y Chavarría (2001), que por la naturaleza de sus funciones, dichas concepciones son diferentes, mas no excluyentes.

La dirección básicamente se refiere a la realización de funciones normativas y operativas; por su parte, el liderazgo está fuertemente asociado a la toma de decisiones y la capacidad de influir sobre las interacciones humanas.

Los citados autores consideran ambos términos complementarios, necesarios e interdependientes. Por otra parte, afirman: "no es posible el ejercicio del uno sin lo otro. Los líderes deben estar capacitados para dirigir y los directores no pueden serlo si no lideran. El liderazgo sin la dirección es imposible y la dirección sin liderazgo sería irresponsable.” (Borrel y Chavarría 2001, p. 19). Una dirección sin liderazgo carece de legitimidad y sostenibilidad en sus acciones.

Con respecto al concepto de gestión, Pozner (2000, p. 16) se refiere a la gestión como:

Un conjunto de procesos teórico-prácticos integrados horizontal y verticalmente dentro del sistema educativo, para cumplir los mandatos sociales. La gestión educativa puede entenderse como las acciones desarrolladas por los gestores que pilotean espacios organizacionales. Es un saber de síntesis capaz de ligar conocimiento y acción, ética y eficacia, política y administración en procesos que tienden al mejoramiento continuo de las prácticas educativas; a la exploración y explotación de todas las posibilidades y a la innovación permanente como proceso sistemático.

Un líder, además de dirigir, gestiona $\mathrm{y}$ ofrece las condiciones para que quienes le acompañan en la conducción de la organización educativa concreten las funciones de índole cotidiana para ocuparse de las funciones macro; para ello se requiere poseer una serie de características como la energía, la asertividad, la habilidad para tomar iniciativas, la apertura a nuevas ideas, la tolerancia a la ambigüedad, el sentido del humor, la habilidad analítica y una posición práctica frente a la vida (Smit y Andrews, citados por Borrel y Chavarría, 2001).

Además de estas las características anteriormente mencionadas, un líder debe poseer ciertas capacidades (ver tabla 2 ).

Las características y capacidades de liderazgo permiten llegar a una clasificación del liderazgo según se detalla en la tabla 3 (ver tabla 3).

El éxito de una gestión estratégica en educación sustentada en un acertado liderazgo será viable si se tiene claro que hoy día las organizaciones educativas deben manejarse dentro de una concepción 
Tabla 2

Otras capacidades de un líder

\begin{tabular}{|l|l|}
\hline Capacidades de un líder & Vivencia en el centro educativo \\
\hline $\begin{array}{l}\text { Capacidad cognitiva: es la habilidad para sintetizar } \\
\text { a información en forma comprensible para los } \\
\text { demás. }\end{array}$ & $\begin{array}{l}\text { Le ofrece a sus docentes información sobre su } \\
\text { trabajo. } \\
\text { La comunicación debe darse en forma acertiva } \\
\text { en todas las direcciones. }\end{array}$ \\
\hline $\begin{array}{l}\text { Capacidades de interacción: habilidad para reconocer } \\
\text { que el éxito depende de la capacidad de trabajar en } \\
\text { equipo y de seleccionar el equipo eficaz. }\end{array}$ & $\begin{array}{l}\text { Elprofesoradoypersonaladministrativotrabajan } \\
\text { en forma conjunta por un proyecto de calidad } \\
\text { consecuente con los objetivos organizacionales. } \\
\text { La interacción debe ser armoniosa el desarrollo } \\
\text { profesional es prioritario, pues los logros } \\
\text { dependen de la capacidad de cada individuo. }\end{array}$ \\
\hline $\begin{array}{l}\text { Capacidades de innovación: habilidad para aceptar el } \\
\text { cambio y asumir riesgos; se acompaña de la habilidad } \\
\text { del aprendizaje en aras de nuevos paradigmas. }\end{array}$ & $\begin{array}{l}\text { Se parte de que el éxito de la organización } \\
\text { requiere cambios de mentalidades de sus } \\
\text { integrantes en el momento oportuno. }\end{array}$ \\
\hline $\begin{array}{l}\text { Capacidades motivacionales: habilidad para } \\
\text { interesar al personal entorno a los distintos } \\
\text { proyectos. }\end{array}$ & $\begin{array}{l}\text { Se considera fundamental incentivar motivación } \\
\text { y energía hacia las metas organizacionales por } \\
\text { convicción. }\end{array}$ \\
\hline
\end{tabular}

Fuente: Tomado de Álvarez (1998) y adaptado por Garbanzo y Orozco.

Tabla 3

Clasificación de liderazgo

\begin{tabular}{|l|l|}
\hline Liderazgo pedagógico & $\begin{array}{l}\text { Centra sus esfuerzos en el proceso de enseñanza-aprendizaje que sucede } \\
\text { en el aula. }\end{array}$ \\
\hline Liderazgo situacional & $\begin{array}{l}\text { Se centra en la gestión de personal, partiendo de la misión del centro } \\
\text { en funciów situaciones diversas del centro educativo y lo asume con una } \\
\text { visión de transformación. Requiere inteligencia para delegar y conducir } \\
\text { a su gente. }\end{array}$ \\
\hline Liderazgo transformador & $\begin{array}{l}\text { Se centra en incorporar al centro educativo la gestión de calidad } \\
\text { transformandolacultura delcentropara estos propósitosy transformándola } \\
\text { hacia procesos de mejora sostenibles. }\end{array}$ \\
\hline Liderazgo técnico & $\begin{array}{l}\text { Se centra en evolucionar en procesos más favorables que los existentes. } \\
\text { Requiere comprometer al profesorado para ejecutar acciones en beneficio } \\
\text { de su propio crecimiento no usualmente ejecutadas y se fomenta la vida } \\
\text { académica. Se le conoce como el liderazgo de la paciencia, puesto que los } \\
\text { resultados en los centros educativos requieren tiempo. }\end{array}$ \\
\hline $\begin{array}{l}\text { Se centra en técnicas y habilidades fundamentales para dirigir } \\
\text { responsablemente el centro educativo. No se requiere exigir más sino } \\
\text { trabajar mejor. }\end{array}$ \\
\hline
\end{tabular}

Fuente: Tomado de Rey y Santa María (2000) y adaptado por Garbanzo y Orozco. 
futurista, propositiva, flexible y responsable, donde la capacidad de adaptarse a las exigencias contextuales con innovadoras propuestas de gestión sean la característica que haga la diferencia en las organizaciones educativas de la postmodernidad; esto con una visión pedagógica y alejadas de la gestiones tradicionales sustentadas en rutinas administrativas, que lejos de formar destruyen los ideales de la comunidad educativa. Esta veterana práctica de administrar, caracterizada por rutinas administrativas formales y rígidas con que han sido tratadas las organizaciones educativas, sin comprender por muchos hasta la fecha que los centros educativos son la unidad de organización clave de los sistemas educativos en aras de la construcción de una sociedad digna para todos.

Los modelos tradicionales de administración escolar resultan, a todas luces, insuficientes para trabajar sobre los problemas en el contexto de sociedades cada vez más complejas, diferenciadas y exigen- tes de calidad y pertinencia educativa. Es imprescindible, afrontar el desafío de promover que lo medular de las organizaciones educativas sea generar aprendizajes bajo un esquema de organización en el que se conjuguen elementos que contribuyan cada vez más a consolidar las organizaciones en función de objetivos de calidad. Es así que un modelo estratégico de gestión debe al menos contener:

1. énfasis en lo pedagógico,

2. nuevas competencias y profesionalización,

3. trabajo en equipo,

4. cultura organizacional con visión de futuro,

5. apertura al aprendizaje y la innovación, $\mathrm{y}$

6. asesoramiento y profesionalización.

Todos estos elementos poseen una relación correlacionada entre cada una de sus partes tal y como se ilustra en la figura 1 :

Figura 1

Componentes fundamentales de un modelo de gestión educativa competitiva
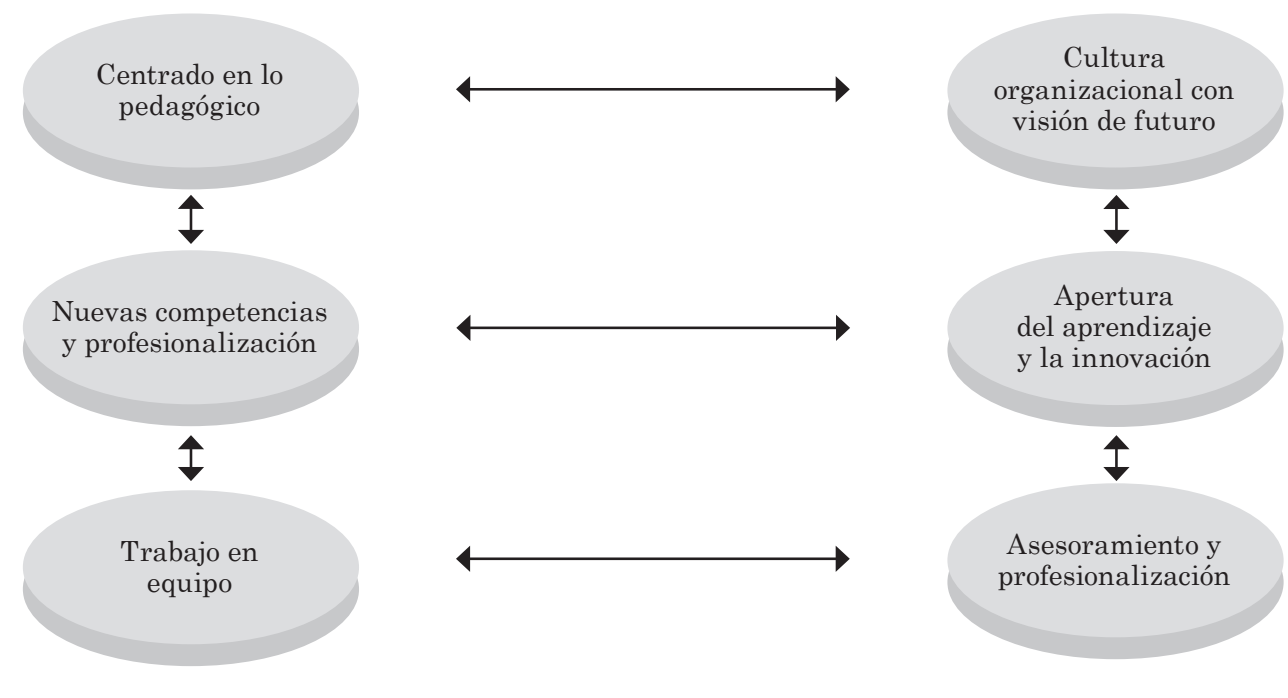

Fuente: Elaboración propia. 
Para que los componentes fundamentales de este modelo de gestión se cumplan se hace imprescindible que los administradores de la educación en sus prácticas laborales aseguren las siguientes funciones directivas (ver tabla 4):

Tabla 4

Funciones directivas de la Administración de la Educación

\begin{tabular}{|l|l|}
\hline$\cdot$ Analizar y sintetizar & $\cdot$ Anticipar y proyectar \\
\hline$\cdot$ Concertar y asociar & $\cdot$ Decidir y desarrollar \\
\hline$\cdot$ Comunicar y coordinar & $\cdot$ Liderar y animar \\
\hline$\cdot$ Evaluar y reenfocar & \\
\hline
\end{tabular}

Fuente: Elaboración propia.

En la medida que el administrador de la educación sea consciente de la importancia de poseer estas funciones directivas, estará en condiciones de asumir una serie de requerimientos de su ejercicio profesional como los que se ilustran la tabla 5:

Tabla 5

Requerimientos en el ejercicio profesional en el campo de la Administración de la Educación

\begin{tabular}{|l|l|}
\hline $\begin{array}{l}\text { un enfoque claro e } \\
\text { interrelacionado }\end{array}$ & $\begin{array}{l}\text { alta capacidad de } \\
\text { concentración }\end{array}$ \\
\hline $\begin{array}{l}\text { conciencia de que los } \\
\text { tiempos de gestión } \\
\text { suponen períodos largos } \\
\text { de gestación }\end{array}$ & $\begin{array}{l}\text { exploración } \\
\text { permanente de las } \\
\text { oportunidades }\end{array}$ \\
\hline $\begin{array}{l}\text { capacidad de } \\
\text { experimentar }\end{array}$ & $\begin{array}{l}\text { disciplina para el } \\
\text { aprendizaje profundo }\end{array}$ \\
\hline gusto por el riesgo & $\cdot$ confianza en sí mismo \\
\hline $\begin{array}{l}\text { sentido de la } \\
\text { responsabilidad }\end{array}$ & $\begin{array}{l}\text { saber aprender } \\
\text { (metaconocimiento) }\end{array}$ \\
\hline compromiso e iniciativa & $\begin{array}{l}\text { saberes y prácticas de } \\
\text { colaboración }\end{array}$ \\
\hline$\cdot$ profesionalidad & $\bullet$ voluntad de servicio \\
\hline
\end{tabular}

Fuente: Elaboración propia.
Estas condiciones se refuerzan acompañadas de la capacidad de asumir el riesgo en forma permanente, de una despolitización del debate educativo, de adecuados procesos de control de calidad, de promoción de la participación de la comunidad, de posicionamiento de la autonomía organizacional y de adecuados procesos de comunicación y toma de decisiones entre las estructuras involucradas. Autores como Estruch (2002) consideran que la evaluación permanente de la gestión directiva y del centro es una condición fundamental. También, es impostergable la capacidad de conocer y comprender el escenario bajo el cual se actúa y los objetivos hacia donde se marcha.

\section{Consideraciones finales}

La gestión moderna de la educación se caracteriza por un liderazgo centrado en lo pedagógico, así como en las nuevas competencias profesionales de su ejercicio. También la cultura organizacional con visión de futuro, el trabajo en equipo, la constante apertura al aprendizaje y la innovación son pilares que marcan su orientación hacia la consecución de los objetivos y fines de la educación.

El liderazgo en una gestión moderna de la educación siempre reconoce las exigencias que el entorno le demanda, articula el proyecto educativo con las necesidades y oportunidades nacionales, transformando así la educación en un instrumento pertinente de cohesión social y movilidad. Esta gestión le presta particular atención a las variables que interactúan en la consecución del proceso de enseñanza-aprendizaje en forma integrada, con una visión principalmente macro del resultado educativo.

El nuevo contexto tecnológico también obliga a la administración de la educación a reinterpretarse como profesión, de manera que le corresponde asumir en forma estratégica e indispensable los retos en los que la educación se ve insertada 
dentro de la contingencia cultural y la era digital, marcos de acción de la gestión moderna de la educación. Debe considerar la gestión moderna de la educación en la conducción de las organizaciones educativas los impactos político, económico, cultural y social que intervienen para que el proceso educativo aspire a ser de calidad y equidad, en el cual la educación inclusiva y la interculturalidad se manifiesten dentro de sus prioridades.

El liderazgo de la gestión moderna de la educación comprende que debe centrarse en lo pedagógico y unir esfuerzos para que el modelo pedagógico que prevalezca se oriente a la construcción del conocimiento, con una visión integral y participativa, y además sus esfuerzos se orienten para alejarse tanto de las rutinas administrativas como del modelo pedagógico tradicionaltransmisionista y conductista, que mucho daño le han hecho a la educación.

No importa cuál sea el énfasis del liderazgo, lo que es determinante como producto de su acción se refleja entre otras cosas en una marcada orientación hacia el aumento permanente de la calidad de la educación, visión de futuro, proactividad, toma de decisiones relevantes para la organización educativa, impulso y ejecución de acuerdos, asesoría y orientación general del proceso educativo, evaluación del proceso como tal, promoción de una cultura permanente de autoevaluación y, por supuesto, la búsqueda constante de procesos de mejora. Todo ello aspira a la consecución de los fines y objetivos de la educación costarricense.

\section{Referencias bibliográficas}

Aguerrondo, I. Lugo, M. T., Rossi, M. (2001). La gestión de la escuela y el diseño de proyectos institucionales. Buenos Aires: Universidad Nacional de Quilmes.
Álvarez, M. (1998). El liderazgo de la calidad total. Madrid: Editorial Escuela Española S.A.

Antúnez, S. (1999). El trabajo en equipo de los profesores y profesoras: factor de calidad, necesidad y problema. El papel de los directivos escolares. Educar, 24, 89-110. Extraído el 09 Setiembre, 2009 de www.raco.cat/ index.php/educar/article/viewPDFInterstitial/20705/20545

Borrell, E. y Chavarría, X. (2001). La planificación y autoevaluación del trabajo de los directivos de los centros docentes. Barcelona: Cisspraxis, S.A.

Braslavsky, C. (2006). Diez factores para una educación de calidad para todos en el siglo XXI. Revista Electrónica Iberoamericana sobre Calidad, Eficacia y Cambio en Educación, 2e(4), 84-101. Extraído el 09 setiembre, 2009 de http://www.rinace.net/ arts/vol4num2e/art5.pdf

Castells, M. (2002). La era de la información: economía, sociedad y cultura (2a ed.). Madrid: Alianza Editorial.

Chiavenato, I. (2004). Introducción a la teoría general de la administración (7 $7^{\mathrm{a}}$ ed.). México: McGraw-Hill.

CONARE/Programa Estado de la Nación en Desarrollo Humano Sostenible. (2005). Informe Estado de la Educación Costarricense (1). San José, Costa Rica: Autor. Costa Rica. Ministerio de Educación Pública. (2006). Boletín de Supervisión Nacional. Ley General de educación Común. Ley Fundamental de Educación. División de Control de Calidad y Macroevaluación del Sistema Educativo Costarricense. San José, Costa Rica: Departamento de Supervisión Nacional. 
Crovi, D. (2002). Sociedad de la Información y el conocimiento. Entre el optimismo y la desesperanza. Revista Mexicana de Ciencias políticas y sociales, XLV. (185), 13-33. Extraído el 01 setiembre, 2009 de http://redalyc.uaemex.mx/ redalyc/src/inicio/IndArtRev.jsp?iCv eNumRev $=4431 \& i$ CveEntRev $=421$

Estruch, J. (2002). Dirección profesional y calidad educativa. Barcelona: CISSPRAXIS.

Garbanzo, G. M. (2004). Necesidades profesionales y de capacitación de los administradores educativos para responder a las demandas del siglo $X X I$. (Informe de Investigación, Escuela de Administración Educativa). (Sin publicar). San José, Universidad de Costa Rica, Escuela de Administración educativa de la Facultad de Educación.

Garbanzo, G. M. y Orozco, V. H. (2007). Desafíos del sistema educativo costarricense: un nuevo paradigma de la administración de la educación. Revista Educación, 31(2), 95-110.
García, N., Rojas, M. y Campos, N. (2002). Administración escolar para el cambio y el mejoramiento de las instituciones educativas. San José, C.R.: Editorial Universidad de Costa Rica.

Gore, Al. (2007). Una verdad incómoda. La crisis planetaria del calentamiento global y cómo afrontarla. Barcelona: Editorial Gedisa.

Menezes, C. (2000, noviembre). Desarrollo de la Sociedad de la información en América Latina y el Cariabe. Ponencia presentada en la Tercera Cumbre sobre la Información del Agua, USA. Montevideo: Biblioteca Virtual. Extraído el 23 abril, 2008 de http:// www.UNESCO.org.uy.informática/ publicaciones/WISpaper_esp.pdf.

Pozner, Pilar (2000). Diez módulos destinados a los responsables de los procesos detransformación educativa. Buenos Aires: IIPE Buenos AiresUNESCO.

Rey,R.y Santa María,J. (2000). Transformar la educción en un contrato de calidad. Barcelona: CISSPRAXIS. 
